12

\title{
Эффекты, связанные с ограниченной геометрией, в нанокомпозитах на основе мезопористых матриц $2 D$-SBA-15 и 3D-SBA-15, содержащих наночастицы нитрита натрия
}

\author{
(C) А.А. Набережнов, ${ }^{1}$ Е.В. Стукова, ${ }^{2}$ О.А. Алексеева, ${ }^{3}$ C.A. Новикова, ${ }^{4}$ A. Franz ${ }^{5}$ \\ ${ }^{1}$ Физико-технический институт им. А.Ф. Иофрер РАН, \\ 194021 Санкт-Петербург, Россия \\ ${ }^{2}$ Амурский государственный университет, \\ 675027 Благовещенск, Амурская обл., Россия \\ ${ }^{3}$ Санкт-Петербургский политехнический университет Петра Великого, \\ 195251, Санкт-Петербург, Россия \\ ${ }^{4}$ Институт химии и химической технологии СО РАН, \\ 660036, Красноярск, Россия \\ ${ }^{5}$ Helmholtz Zentrum Berlin, Hahn Meitner Platz 1, \\ 14109 Berlin, Germany \\ e-mail: alex.nabereznov@mail.ioffe.ru
}

Поступило в Редакцию 24 мая 2019 г.

В окончательной редакции 24 мая 2019 г.

Принято к публикации 10 июня

\begin{abstract}
Из анализа температурной эволюции дифракционных спектров рассеяния нейтронов на композитах, полученных при введении нитрита натрия в поры мезопористых матриц $2 D$-SBA-15 (средний диаметр пор 69(4) $\AA$ ) и 3D-SBA-15 (средний диаметр пор 94(5) А), получены температурные зависимости сегнетоэлектрического параметра порядка для наноструктурированного нитрита натрия при нагреве и охлаждении. Показано, что при нагреве для обоих нанокомпозитных материалов фазовый переход в параэлектрическую фазу происходит при $T_{C}=433 \pm 1 \mathrm{~K}$. Определены размеры наночастиц в обоих нанокомпозитах и установлено, что их размер уменьшается при нагреве при приближении к температуре сегнетоэлектрического фазового перехода. Обнаружен температурный гистерезис $(\sim 15-20 \mathrm{~K})$ в температурной зависимости параметра порядка между режимами нагрева и охлаждения.
\end{abstract}

Ключевые слова: ограниченная геометрия, нанокомпозитные материалы, дифракция нейтронов, фазовые переходы.

DOI: $10.21883 /$ JTF.2019.12.48497.207-19

\section{Введение}

Массивный нитрит натрия принадлежит к семейству сегнетоэлектриков типа „порядок-беспорядок“, наличие спонтанной поляризации, направленной вдоль оси $\mathbf{b}$, связано с упорядочением в сегнетоэлектрической фазе групп $\mathrm{NO}_{2}$, сопровождаемым смещением ионов натрия. В высокотемпературной фазе соответствующие кристаллографически эквивалентные позиции заселены равновероятно. Физической реализацией параметра порядка $\eta$ в этом соединении в сегнетоэлектрической фазе является разность $\eta=f_{1}-f_{2}$ [1], где $f_{1}$ и $f_{2}-$ заселенности вышеупомянутых позиций групп $\mathrm{NO}_{2}$. При низких температурах и вплоть до $\sim 436 \mathrm{~K}$ массивный нитрит натрия находится в сегнетоэлектрической фазе $(a=3.57 \AA, b=5.578 \AA, c=5.39 \AA)$ с орторомбической симметрией (пространственная группа $\operatorname{Im} 2 m$ ), в области $436-437 \mathrm{~K}$ существует несоразмерная фаза, а выше $437 \mathrm{~K} \quad \mathrm{NaNO}_{2}$ переходит в параэлектрическую фазу с пространственной группой Immm. Ранее было показано, что для нанокомпозитных материалов (НКМ), содержащих наночастицы $\mathrm{NaNO}_{2}$ и полученных при внедрении последнего в пористые матрицы (боросиликатные стекла [2-4], искусственные опалы [5,6] и каналы хризотилового асбеста [7]), наблюдается значительная модификация макроскопических свойств (в том числе и температурной зависимости параметра порядка) при уменьшении характерного среднего диаметра пор и соответственно размера наночастиц. Так, в работе [8] был установлен критический размер (около 50-55 nm) наночастиц нитрита натрия в пористых стеклах (Porous Glasses - PG), меньше которого наблюдается смена фазового перехода (ФП) первого рода, характерного для массивного материала, на ФП второго рода, который наблюдался в НКМ с $\mathrm{NaNO}_{2}$ для пористых стекол со средним диаметром пор 3 и $7 \mathrm{~nm}$. Мезопористые силикатные матрицы типа SBA-15 впервые были получены в работе [9] и представляли собой массив двумерных гексагонально-упорядоченных (пространственная группа $P 6 \mathrm{~mm}$ ) каналов. Диаметр канала можно варьировать управляемым образом в диапазоне 40-300 , причем дисперсия диаметров оказывается весьма небольшой. Позднее [10,11] были получены 3D-матрицы SBA-15, в которых симметрия каналов является кубической (про- 
странственная группа $I a \overline{3} d)$. Было также показано [12], что в $3 D$-SBA-15 существуют 2 типа пор: гексагональноупорядоченные каналы, локально разупорядоченные и случайно распределенные слившиеся поры. При увеличении температуры гидротермической обработки размер упорядоченных каналов изменяется слабо, в то время как характерный размер слившихся пор и вклад этой фракции увеличивается и, в конце концов, становится преобладающим. Следует отметить, что матрицы SBA и PG состоят в основном из диоксида кремния и отличаются только топологией пор: в первом случае мы имеем дело с регулярной системой пор, а во втором со случайной $3 D$-дендритной системой взаимосвязанных пор, поэтому можно ожидать общности эффектов (при одинаковых размерах внедренных наночастиц), связанных с влиянием ограниченной геометрии на свойства внедренных материалов. Были проведены исследования [13] температурной и частотной зависимостей диэлектрического отклика НКМ „нитрит натрия $+\mathrm{SBA}^{\text {“ и и }}$ показано, что для наночастиц нитрита натрия в матрицах SBA значения действительной части диэлектрической проницаемости $\mathcal{E}^{\prime}$ на низких частотах примерно в 10 раз больше, чем для массивного материала, что, в целом, соответствует данным, полученным для НКМ на основе пористых стекол, содержащих наночастицы $\mathrm{NaNO}_{2}[2,5,8]$. В то же время структурных исследований НКМ на основе матриц SBA ранее не проводилось, и особенности температурной эволюции структуры оставались неизученными. Следует также отметить, что средние диаметры пор (для $2 D$-SBA-15 - $69 \pm 4 \AA$, для $3 D$-SBA-15 - $94 \pm 5 \AA$ ) хорошо соответствуют средним диаметрам пор в PG, на основе которых были получены $\mathrm{HKM} \mathrm{PG}+\mathrm{NaNO}_{2}$ и для которых наблюдались изменения рода сегнетоэлектрического фазового перехода $[3,8]$, резкий рост действительной и мнимой части диэлектрического отклика в параэлектрической фазе $[2,5]$ и другие аномалии макроскопических свойств при уменьшении диаметра каналов в пористых матрицах, в частности в пористых стеклах, хризотиловых асбестах и искусственных опалах [4-8,14-17].

\section{1. Приготовление образцов и эксперимент}

Матрицы были изготовлены в ИХХТ СО РАН по технологии, близкой к изложенной в работе [18]. В использованных нами матрицах $2 D$-SBA-15 представляли собой гранулы с гексагонально-упорядоченными системами $1 D$-каналов с диаметром $69 \pm 4 \AA$, толщина стенок между каналами $-33.7 \pm 2.0 \AA$, а параметр ячейки гексагональной упаковки составлял $102.8 \pm 6.2 \AA$. $3 D$ SBA-15 отличались тем, что в стенках $1 D$-каналов имеются субмезопоры („разрывы“ со средним диаметром около $20 \AA$ ) [13]. Диаметр канала для этих матриц составлял $94 \pm 5 \AA$, толщина стенок 28(2) $\AA$, а параметр ячейки $-122.6 \pm 7.4 \AA$. Внедрение сегнетоэлектрика
$\mathrm{NaNO}_{2}$ в поры наноразмерных силикатных матриц проводилось из водного раствора в АмГУ. Сегнетоэлектрик растворялся в дистиллированной воде до получения насыщенного раствора. Порошок мезопористого вещества засыпался в раствор так, чтобы весь раствор проник в поры, после чего смесь высушивалась. Данная процедура выполнялась 3 раза для повышения коэффициента заполнения пор сегнетоэлектриком. Контроль степени заполнения проводился с помощью взвешивания образцов до и после заполнения. Степень заполнения составляла $60-70 \%$. Измерения температурной эволюции кристаллической структуры HKM SBA-15 $+\mathrm{NaNO}_{2}$ проводились на нейтронном дифрактометре высокого разрешения Е9 (реактор BER II, Helmholtz Zentrum Berlin, Германия) при нагреве и охлаждении. Измерения дифракционных спектров массивного нитрита натрия (для уточнения инструментальной функции разрешения) и пустых пористых матриц проводились только при комнатной температуре. Длина волны падающих нейтронов была $1.798 \AA$, температурный интервал измерений $\mathrm{RT}-450 \mathrm{~K}$ при нагреве и $450-380 \mathrm{~K}$ при охлаждении, т.е. выше и ниже температуры сегнетоэлектрического фазового перехода. Стабильность поддержания температуры во время измерений была не хуже $\pm 0.5 \mathrm{~K}$. Измерение одной температурной точки занимало от 4 до 8 часов экспериментального времени.

\section{2. Результаты и обсуждение}

При всех температурах на дифракционных спектрах обоих НКМ наблюдаются только упругие пики, соответствующие кристаллической структуре нитрита натрия, и диффузный фон от рассеяния на аморфном $\mathrm{SiO}_{2}$ самой матрицы (рис. 1). Параметры решетки для наночастиц $\mathrm{NaNO}_{2}$ при $300 \mathrm{~K}$ практически совпадают с данными, известными для массивного нитрита натрия [1,19], од-

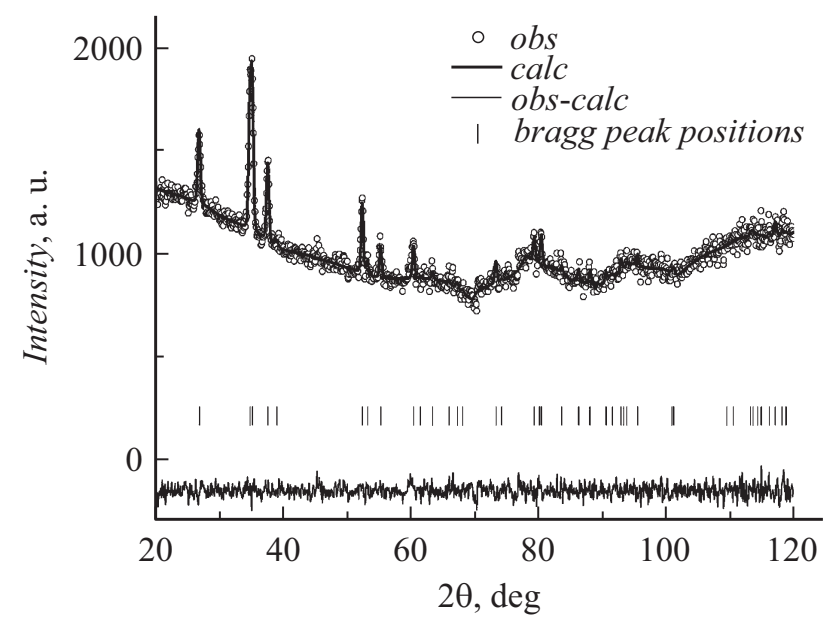

Рис. 1. Дифракционный спектр для $\mathrm{HKM} 3 D-\mathrm{SBA}-15+\mathrm{NaNO}_{2}$ при 300 К. Белые точки („obs“) - эксперимент, черная линия („calc“) подгонка, нижняя линия („obs-calc“) - невязка, вертикальные линии - положения упругих пиков. 


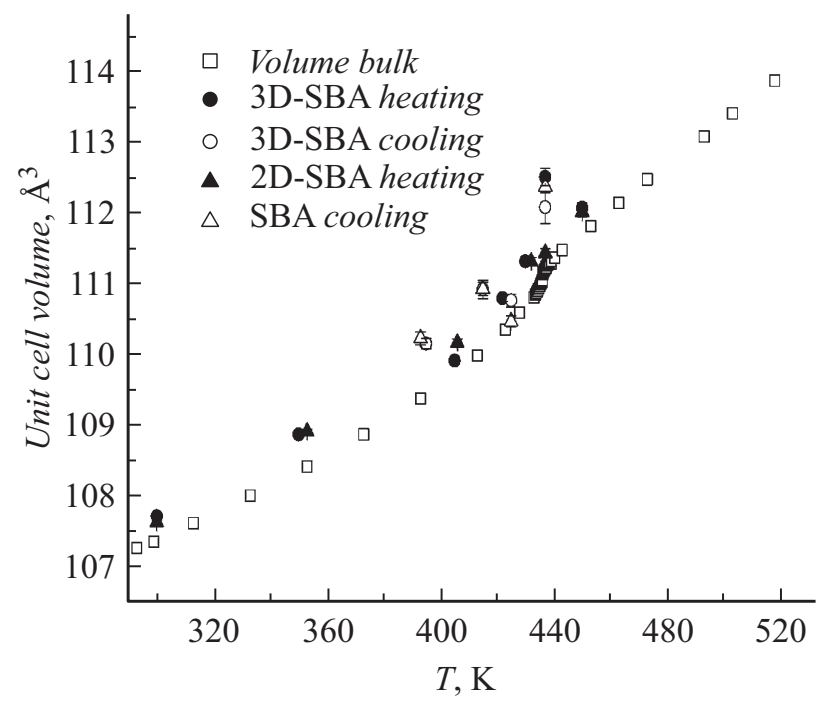

Рис. 2. Температурные зависимости объемов элементарной ячейки для массивного $\mathrm{NaNO}_{2}$ (белые квадраты) [19] и НКМ: черные символы - нагрев, белые символы - охлаждение.

нако при повышении температуры параметры а и b становятся несколько больше. Эта тенденция сохранялась вплоть до перехода в параэлектрическую фазу. При охлаждении до $380 \mathrm{~K}$ температурная зависимость а и b практически совпадала с наблюдаемой при нагреве образца. Существенной разницы в температурных зависимостях параметров ячейки для НКМ на основе $2 D$-SBA-15 и 3D-SBA-15 не обнаружено. Температурная зависимости объема элементарной ячейки для обоих НКМ и массивного материала (из работы [20]) приведены на рис. 2. На рис. 2 хорошо видно, что как для массивного, так и для наноструктурированного нитрита натрия наблюдается аномалия вблизи температуры сегнетоэлектрического фазового перехода. Означает ли это, что наночастицы $\mathrm{NaNO}_{2}$ в данных матрицах SBA сохраняют свойства массивного материала, или же этот эффект связан с тем, что значительная часть нитрита натрия при изготовлении образца кристаллизуется не в порах матриц, а в виде достаточно больших по размеру кристаллитов, например, на поверхности гранул SBA или в пространстве между гранулами? Проанализируем сначала форму линии упругих отражений. Для массивного материала форма линии должна практически соответствовать функции разрешения (инструментальной функции) дифрактометра, а для наночастиц должно наблюдаться уширение линии из-за размерного эффекта $[21,22]$ и возможного наличия упругих напряжений. В случае смеси массивного и наноструктурированного нитрита натрия в образце форма линии упругих пиков должна искажаться, и из этого искажения можно определить величину примеси массивного (или ультрадиспергированного) нитрита натрия. Так как уширение из-за размерного эффекта в целом пропорционально $1 / \cos \theta$, а из-за упругих напряжений $\sim \operatorname{tg} \theta$ (здесь $\theta-$ угол рас- сеяния), то для анализа были выбраны упругие пики при достаточно малых $\theta$, где вкладом упругих напряжений можно пренебречь. На рис. 3 в качестве примера приведены отнормированные интенсивности упругого пика (020) для НКМ 3D-SBA-15 + $\mathrm{NaNO}_{2}$ (сплошная линия) и для массивного нитрита натрия (штриховая линия „instrumental resolution“) при комнатной температуре. Хорошо видно, что пик (020) для НКМ явно шире, чем для массивного материала, как и должно быть для частиц нанометрового масштаба.

На следующем этапе из анализа дифракционных спектров были получены значения средних размеров наночастиц NaNO2, внедренных в пористые матрицы 2D-SBA и 3D-SBA, температурные зависимости которых приведены на рис. 4. Отметим, что средний размер частиц ( 65-70 nm при комнатной температуре) значительно превышает диаметры каналов пористых матриц (диаметр канала $\sim 10 \mathrm{~nm})$. Таким образом, можно ожидать, что нитрит натрия в каналах формирует достаточно протяженные нанонити, т.е. наночастицы существенно анизотропны, а это, в свою очередь, может приводить к появлению преимущественной ориентации нанокристаллитов. В этом случае в эксперименте должно наблюдаться разное уширение дифракционных пиков с различными индексами Миллера, как это было показано на примере наночастиц свинца в пористых стеклах [23]. Проведенный анализ ширин дифракционных спектров с разными $h k l$ показал, что такого эффекта для $3 D$-SBA15 не наблюдается. Таким образом, можно утверждать, что в матрице $3 D$-SBA-15 наночастицы формируются в нескольких соседних каналах, так как существует коалесценция каналов, как показано для этой матрицы в работе [18]. В случае $2 D$-SBA-15 провести подобный анализ мы не смогли из-за недостаточной статистики.

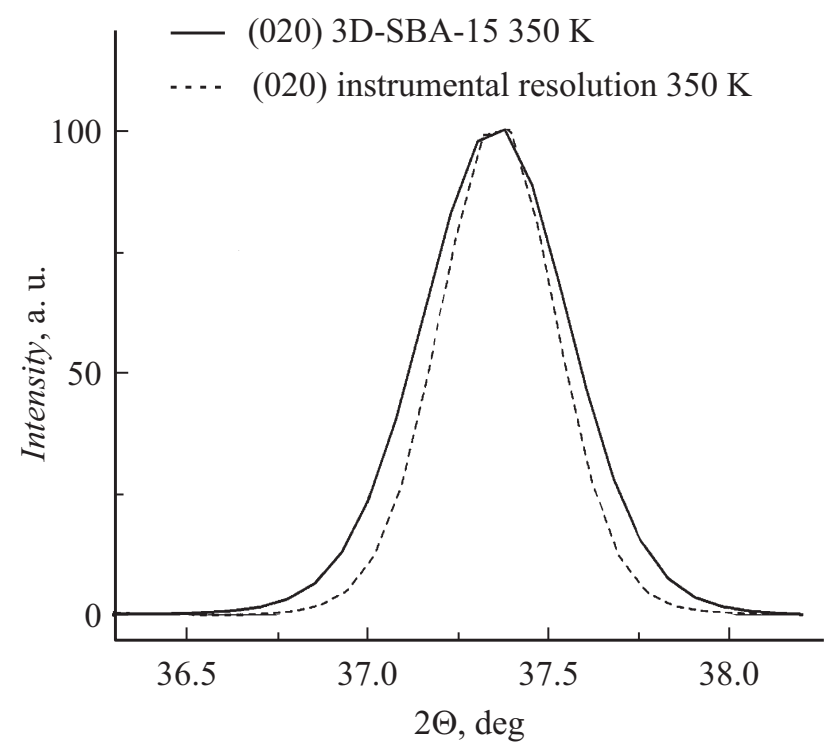

Рис. 3. Форма линии упругого пика $(020)$ для НКМ $3 D$-SBA$15+\mathrm{NaNO}_{2}$ (черная кривая) и инструментальное разрешение (штриховая линия) при комнатной температуре. 


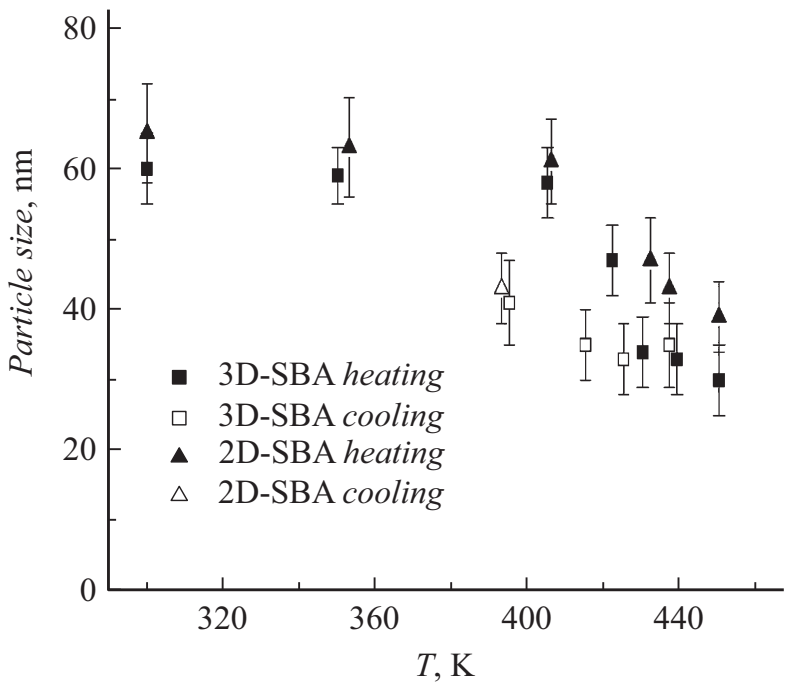

Рис. 4. Температурные зависимости размеров наночастиц нитрита натрия в НКМ $2 D$-SBA- $15+\mathrm{NaNO}_{2}$ (треугольники) и $3 D$-SBA-15 + $\mathrm{NaNO}_{2}$ (квадраты). Черные символы - нагрев, белые символы - охлаждение.

При повышении температуры происходит уменышение дифракционного размера частиц, причем наиболее резкий спад начинается в районе температуры $400 \mathrm{~K}$, а вблизи температуры фазового перехода в параэлектрическую фазу происходит значительное (на 30-50\%) уменьшение среднего диаметра наночастиц. Рассмотрим возможные микроскопические механизмы этого явления. Первый их них связан с понижением температуры плавления $T_{\text {melt }}$ при уменьшении размера частиц $[24,25]$, причем это понижение может достигать 100-200 K [26]. В случае массивного нитрита натрия температура плавления составляет около $554 \mathrm{~K}$, и, в принципе, можно ожидать существенного понижения $T_{\text {melt }}$ в условиях ограниченной геометрии. В то же время такое значительное уменьшение размера (на 30-50\%) должно было бы привести к явно выраженному уменьшению интенсивности упругих пиков на дифракционных спектрах, так как интенсивность пропорциональна объему кристаллического материала, а рассеяние на расплавленном нитрите натрия привело бы к появлению некоторого дополнительного фона, структурированного вблизи этих упругих пиков. В эксперименте такого явления мы не наблюдали, и поэтому предположение о возможном „размерном“ плавлении следует отвергнуть. Второй механизм - это появление внутренних разрывов (в частности в перемычках, соединяющих соседние каналы при коалесценции) в самих наночастицах при нагреве. Причиной появления разрывов может быть сжатие самой матрицы при нагреве, так как SBA-15 в диапазоне 298-1573 K имеет большой отрицательный коэффициент теплового расширения (КТР): $\alpha_{a}=-4.3 \cdot 10^{-6} \mathrm{~K}^{-1}[27]$. При охлаждении из параэлектрической фазы происходит расширение матрицы и наблюдается тенденция восстановления размера наночастиц (рис. 4), однако недостаток экспериментального времени не позволил нам проследить происходит ли это восстановление полностью при достижении комнатной температуры и за какое время.

Как было показано еще в работе [19], в сегнетоэлектрической фазе $\mathrm{NaNO}_{2}$ существуют два типа (семейства) дифракционных пиков: интенсивности одного из них не зависят от сегнетоэлектрического параметра порядка $\eta$ (например рефлексов (101), (020), (200) в случае дифракции нейтронов), а для другого (например (022), (132)) практически пропорциональны $\eta^{2}$. Используя этот факт, мы получили температурные зависимости $\eta(T)$ для обоих типов НКМ: $2 D-\mathrm{SBA}-15+\mathrm{NaNO}_{2}$ и $3 D$-SBA-15 $+\mathrm{NaNO}_{2}$ (рис. 5); зависимость $\eta(T)$ для массивного нитрита натрия взята из работы [19] и приведена для сравнения. Легко видеть, что при нагреве кривая зависимости $\eta(T)$ для обоих НКМ лежит несколько ниже по сравнению с зависимостью для массивного материала, причем при всех температурах. Разница значений $\eta(T)$ для НКМ и для массивного материала в области температур до 400-410 K может быть связана с существованием поверхностного слоя, в котором сегнетоэлектрическое упорядочение разрушено. Предполагая, что в целом наночастица имеет цилиндрическую форму (или несколько цилиндров, связанных малыми перемычками), можно оценить толщину этого слоя, который составляет 7-9 А, т. е. 1-2 соответствующих параметра ячейки $\mathrm{NaNO}_{2}$. Эта оценка хорошо совпадает с толщиной граничного слоя для НКМ „пористое стекло-нитрит натрия“ в случае пористого стекла со средним диаметром пор $20 \mathrm{~nm}$ [6].

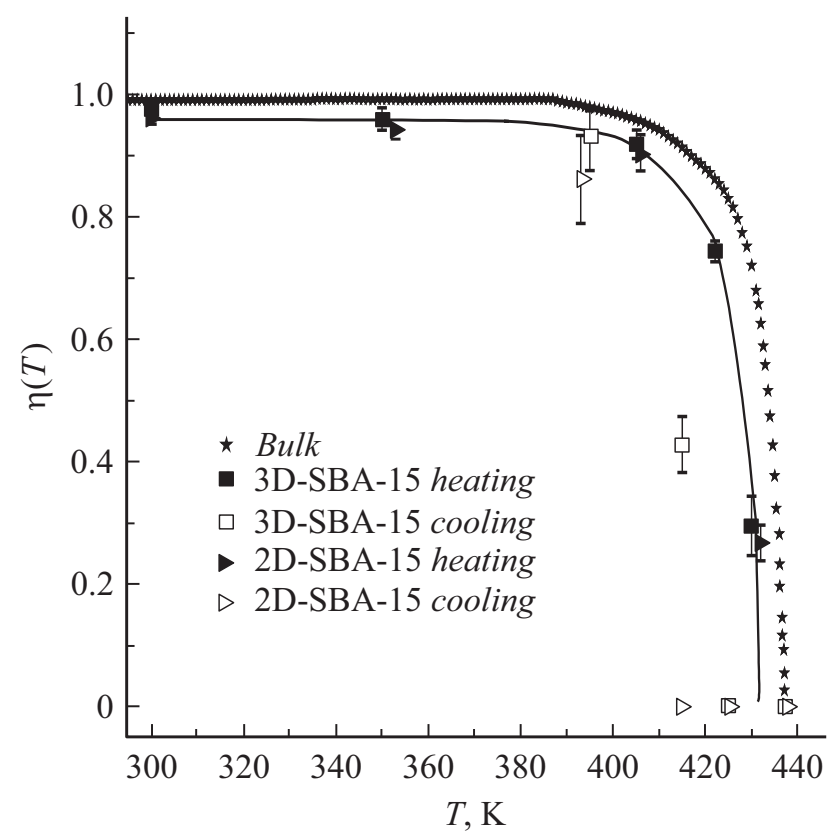

Рис. 5. Температурные зависимости параметра порядка в НКМ 2D-SBA-15+ $\mathrm{NaNO}_{2}$ (треугольники) и $3 D$-SBA$15+\mathrm{NaNO}_{2}$ (квадраты). Черные символы - нагрев, белые символы - охлаждение. Черные звезды - зависимость $\eta(T)$ для массивного $\mathrm{NaNO}_{2}$ [19] 
Из экстраполяции зависимостей $\eta(T)$ при высоких температурах получены оценки температуры Кюри $T_{C}$ в данных НКМ: $T_{C}=433(1) \mathrm{K}$, что совпадает со значением, полученным из данных диэлектрической спектроскопии [13]. Здесь следует обратить внимание на то, что выше $415 \mathrm{~K}$ размер наночастиц нитрита натрия становится меньше критического ( 50-55 nm) [8], для которых $T_{C}$ должна быть ниже $426 \mathrm{~K}$ [3], а сам ФП становится ФП второго рода. В данном эксперименте даже при $430 \mathrm{~K}$ сегнетоэлектрический дальний порядок не исчезает: величина $\eta$ при $430 \mathrm{~K}$ составляет $0.29 \pm 0.05$ для HКM $3 D$-SBA-15 $+\mathrm{NaNO}_{2}$ и $0.27 \pm 0.03$ для НКМ $2 D$ $\mathrm{SBA}-15+\mathrm{NaNO}_{2}$. Наиболее вероятной причиной этого эффекта является влияние матрицы, а именно ее сжатие при повышении температуры. Действительно, согласно фазовой диаграмме „температура-давление“ для $\mathrm{NaNO}_{2}$, приведенной в работе [28], увеличение давления приводит к повышению $T_{C}$. Таким образом в данных НКМ существует конкуренция двух процессов: с одной стороны, уменьшение размера наночастицы должно вести к понижению $T_{C}$, a, с другой стороны, сжатие матрицы должно повышать $T_{C}$, что, в конечном счете, и приводит к величине $T_{C}=433 \mathrm{~K}$. В принципе появление упругих напряжений (или их изменение) в области температуры ФП можно определить из анализа изменения формы линии упругих пиков при больших переданных импульсах $Q$. Имеющиеся в настоящее время данные не позволяют провести подобный анализ, но мы планируем провести более детальные исследования в ближайшее время на дифрактометре высокого разрешения HRFD в ОИЯИ (г. Дубна).

Температурная зависимость $\eta(T)$ в данных НКМ демонстрирует значительный температурный гистерезис: при охлаждении появление сегнетоэлектрической фазы в НKM 3D-SBA-15+ $\mathrm{NaNO}_{2}$ происходит в температурном интервале $415-425 \mathrm{~K}$, в НКМ $2 D-\mathrm{SBA}-15+\mathrm{NaNO}_{2} \quad 400-415 \mathrm{~K}$. Согласно данным [8] о $T_{C}$, для наночастиц $\mathrm{NaNO}_{2}$ со средним размером в диапазоне 30-40 nm (как в наших образцах при охлаждении - рис. 4) при ФП в сегнетоэлектрическое состояние следует ожидать понижения температуры Кюри до $\sim 420 \mathrm{~K}$, что мы и наблюдали для данных НКМ.

Отметим также, что величина температурного гистерезиса в поведении $\eta(T)$ в данных нанокомпозитах составляет около $15-20 \mathrm{~K}$. Ранее значительное увеличение температурного гистерезиса при фазовом переходе в $\mathrm{NaNO}_{2}$ в условиях ограниченной геометрии было обнаружено для НКМ, в которых в качестве пористых матриц были использованы искусственные опалы [4,6] и пористые стекла [29] со средним диаметром пор $46 \mathrm{~nm}$, величины гистерезиса в которых составляли порядка $10 \mathrm{~K}$.

Используя тот факт, что при охлаждении при $T=425 \mathrm{~K}$ параметр порядка $\eta=0$ (ошибка определения этого параметра составляет \pm 0.04$)$, мы определили возможную примесь массивного материала. Действительно, при этой температуре для массивного материала $\eta=0.81$ (рис. 5), тогда вклад в интенсивность пика (022) от массива будет $I \sim V_{\text {mass }} \eta^{2}$, т. е. $0.66 V_{\text {mass }}$; вкладом в интенсивность от наностуктурированного нитрита натрия при этой температуре можно пренебречь в силу его малости. Если относительная примесь массивного нитрита натрия составляет порядка $10 \%$ от массы наноструктурированного $\mathrm{NaNO}_{2}$, то интенсивность отражения (022) для всего НКМ будет (при $425 \mathrm{~K}) \sim 0.066$ от интенсивности рефлекса (022) при комнатной температуре, что, в свою очередь, даст некое фиктивное значение $\eta$ для всего нанокомпозита $\sim 0.25$. Такая величина параметра порядка $\eta$ намного превосходит ошибку ее определения при $425 \mathrm{~K}$ и должна была бы быть зарегистрирована. Таким образом, можно сделать вывод, что примесь массивного нитрита натрия в данных НКМ меньше $10 \%$.

\section{Заключение}

В работе проведено исследование температурной эволюции кристаллической структуры $\mathrm{NaNO}_{2}$, внедренного в нанопористые матрицы $3 D$-SBA-15 и $2 D$-SBA-15 методом дифракции нейтронов. Определены характерные размеры наночастиц $\mathrm{NaNO}_{2}$ в порах матриц и показано, что при повышении температуры выше $410 \mathrm{~K}$ (при приближении к $T_{C}$ ) эти размеры уменьшаются. Наиболее вероятной причиной этого эффекта является сжатие самой матрицы при нагреве. Получены температурные зависимости параметра порядка $\eta(T)$ для данных НКМ и установлено, что при нагреве ФП в параэлектрическую фазу происходит при $T_{C}=433 \pm 1 \mathrm{~K}$. При охлаждении наблюдается значительный температурный гистерезис в зависимости $\eta(T)$ порядка $15-20 \mathrm{~K}$. Установлена роль матрицы в модификации свойств НКМ. Показано, что примесь массивного материала в данных НКМ не превышает 5\%.

\section{Финансирование работы}

Работа выполнена при частичной поддержке РФФИ (грант 19-02-00760). В Санкт-Петербургском политехническом университете Петра Великого исследования выполнялись в рамках тематики проекта 3.1150.2017/4.6. В АмГУ работа выполнена при частичной поддержке проекта Минобрнауки РФ 3.5512.2017/8.9.

\section{Конфликт интересов}

Авторы заявляют, что у них нет конфликта интересов.

\section{Список литературы}

[1] Kay M.I. // Ferroelectrics. 1972. Vol. 4. P. 235.

[2] Colla E.V., Koroleva E.Yu., Kumzerov Yu.A., Savenko B.N., Vakhrushev S.B. // Ferroelectric Lett. 1996. Vol. 20. P. 143. 
[3] Naberezhnov A., Fokin A., Kumzerov Yu, Sotnikov A., Vakhrushev S., Dorner B. // Eur. Phys. J. E. 2003. Vol. 12. P. s21.

[4] Pan'kova S.V., Poborchii V.V., Solov'ev V.G. // J. Phys. Condens. Mat. 1996. Vol. 8. P. L203.

[5] Kinka M., Banys J., Naberezhnov A. // Ferroelectrics. 2007. Vol. 348. P. 67.

[6] Бескровный А.И., Василовский С.Г., Вахрушев С.Б., Курдюков Д.А., Зворыкина О.И., Набережснов А.А., Окунева H.M., Tovar M., Rysiakiewicz-Pasek E., Jagus P. // ФТT. 2010. Т. 52. Вып. 5. С. 1021.

[7] Borisov S., Hansen T., Kumzerov Yu., Naberezhnov A., Simkin V., Smirnov O., Sotnikov A., Tovar M., Vakhrushev S. // Physica B. 2004. Vol. 350. P. E1119.

[8] Fokin A., Kumzerov Yu., Koroleva E., Naberezhnov A., Smirnov O., Tovar M., Vakhrushev S., Glazman M. // J. Electroceram. 2009. Vol. 22. P. 270.

[9] Zhao D., Feng J., Huo Q., Melosh N., Fredrickson G.H., Chmelka B.F., Stucky G.D. // Science. 1998. Vol. 279. P. 548.

[10] Tian B., Liu X., Yu C., Gao F., Luo Q., Xie S., Tu B., Zhao D. // Chem. Commun. 2002. N 11. P. 1186.

[11] Chan Y.-T., Lin H.-P., Mou C.-Y., Liu S.-T. // Chem. Commun. 2002. N 23. P. 2878.

[12] Yuan P., Tan L., Pan D., Guo Y., Zhou L., Yang J., Zou J., Yu. C. // New J. Chem. 2011. Vol. 35. P. 2456.

[13] Ефимова О.В., Стукова Е.В, Королева Е.Ю., Суханов Р.В. // Вестник АмГУ. 2017. Т. 79. С. 165.

[14] Vakhrushev S.B, Kumzerov Yu. A., Fokin A., Naberezhnov A.A., Zalar B., Lebar A., Blinc R. // Phys. Rev. B. 2004. Vol. 70. 132102.

[15] Tien C., Charnaya E.V., Lee M.K., Baryshnikov S.V., Sun S.Y., Michel D., Böhlmann W. // Phys. Rev. B. 2005. Vol. 72. 104105.

[16] Baryshnikov S.V., Tien C., Charnaya E.V., Lee M.K., Michel D., Böhlmann W. // Ferroelectrics. 2008. Vol. 363. P. 177.

[17] Rysiakiewicz-Pasek E., Poprawski R., Polanska J., Urbanowicz A., Sieradzki A. // J. Non-Cryst. Solids. 2006. Vol. 352. P. 4309.

[18] Parfenov V.A., Ponomarenko I.V., Novikova S.A. // Mater. Chem. Phys. 2019. В печати. doi.org/10.1016/j.matchemphys.2019.04.087

[19] da Costa Lamas A., Chang S.-L., Caticha-Ellis S. // Phys. Status Solidi A. 1981. Vol. 68. P. 173.

[20] Kucharczyk D., Pietraszko A., Lukaszewicz K. // Phys. Status Solidi A. 1976. Vol. 37. P. 287.

[21] Thompson P., Cox D., Hastings B. // J. Appl. Phys. 1987. Vol. 20. P. 79.

[22] Langford J.I. // J. Appl. Phys. 1978. Vol. 11. P. 10.

[23] Golosovsky I.V., Delaplane R.G., Naberezhnov A.A., Kumzerov Yu.A. // Phys. Rev. B. 2004. Vol. 69. 132301.

[24] Bertsch G. // Science. 1997. Vol. 277. P. 1619.

[25] Takagi M. // J. Phys. Soc. Jpn. 1954. Vol. 9. P. 359.

[26] Zhang M., Efremov M.Yu., Schiettekatte F., Olson E.A., Kwan A.T., Lai S.L., Wisleder T., Greene J.E., Allen L.H. // Phys. Rev. B. 2000. Vol. 62. N 15. P. 10548.

[27] Shah P., Ramaswamy V. // Micropor. Mesopor. Mat. 2008. Vol. 114. P. 270.

[28] Rapoport E. // J. Chem. Phys. 1966. Vol. 45. N 8. P. 2721.

[29] Rysiakiewicz-Pasek E., Komar J., Ciźman A., Poprawski R. // J. Non-Cryst. Solids. 2010. Vol. 356. P. 661. 